\section{LA BIBLIOTECA LITERARIA DEL ESTUDIANTE}

\author{
Mario Pedrazuela Fuentes \\ Universidad Carlos III de Madrid. Instituto de Historia del Centro de \\ Ciencias Humanas y Sociales del CSIC \\ C/ Meléndez Valdés, 3, Bajo, D, 28015, Madrid \\ adamevas@gmail.com
}

\begin{abstract}
This paper analyzes the repercussion of the Student's Literary Library in the teaching of literature in Bachillerato. The Student's Literary Library was a book collection stimulated by the Instituto-Escuela in order to encourage the great classical authors reading among the bachillerato students. The selection was made with a view to its phylological and literary interest, and so as to make it affordable and respectful with the texts just as they were written. It was supervised by Ramón Menéndez Pidal, and her wife, María Goiry, participated in it, as well as many Center of Historic Studies collaborators and teachers of the InstitutoEscuela itself.
\end{abstract}

KEY WORDS: Literature; Student's Literary Library; School Institute; High school degree; Literature of teaching.

\section{UNA VISIÓN DE LA ENSEÑANZA DE LA LITERATURA A FINALES DEL S. XIX Y PRINCIPIOS DEL XX}

El Real Decreto de 25 de septiembre de 1845 reforma los planes de estudio y organiza el sistema educativo en las etapas que hoy conocemos: primaria, secundaria y superior. En cuanto a la educación secundaria, el plan establecía que la enseñanza de la lengua latina y castellana se impartiera de forma conjunta: "El proyecto establece pues que el estudio del latín no se interrumpa mientras dure la segunda enseñanza, y que a la par se haga el de la lengua patria, que tanto apoyo ha de encontrar"'. No será hasta 1901, con la reforma educativa llevada a cabo por el conde de Romanones ${ }^{2}$, ministro entonces de Instrucción Pública, cuando se separen en dos asignaturas. En la práctica, esta convivencia de las dos lenguas provocaba que los profesores tuvieran tiempo únicamente para explicar la primera de ellas, quedándose la nuestra "a medio hacer 0 sin empezar", como dice el decreto de 1901. De tal modo que el profesor apenas podía enseñar ni la gramática, ni la composición, ni la caligrafía, lo que suponía una preocu-

\section{THE STUDENT'S LITERARY LIBRARY}

RESUMEN: El presente trabajo analiza la repercusión que tuvo la Biblioteca Literaria del Estudiante en la enseñanza de la literatura en el Bachillerato. La Biblioteca Literaria del Estudiante fue una colección de libros promovida desde el Instituto-Escuela para fomentar la lectura entre los estudiantes de bachillerato de los grandes autores clásicos de nuestra literatura. Se trata de una selección basada en su interés filológico y literario, que fuera asequible para los estudiantes y que a la vez respetara los textos tal y como fueron escritos. Dirigida por Ramón Menéndez Pidal, en ella colaboraron, además de su esposa, Maria Goiry, muchos de los investigadores que trabajaron en el Centro de Estudios Históricos, asi como profesores del propio Instituto-Escuela.

PALABRAS CLAVE: Literatura; Biblioteca Literaria del Estudiante; Instituto Escuela; Bachillerato; Enseñanza de la Literatura.

pación, debido al "lamentable desconocimiento del arte de la escritura con que se sale de nuestros institutos", como sigue diciendo el preámbulo. Sin embargo, nada se dice en él sobre la lectura.

En vista del escaso tiempo del que disponía el profesor para explicar la gramática o la caligrafía de la lengua castellana, es de suponer que el que se dedicaba a la lectura fuera todavía más escaso, y el poco que tuvieran lo utilizaran para leer obras en latín y no en nuestra lengua, porque como decía el Decreto de 1845:

Los libros de la antigüedad tienen otra ventaja: el servicio que hacen a la juventud no es solamente literario, sino también moral y filosófico, suministran al paso multitud de conocimientos útiles y provechosos; presentan ejemplos de inclitos hechos y grandes virtudes, nos familiarizan con los personajes más eminentes que ha producido la humanidad en politica, en ciencias, en arte y literatura; en todas las páginas se ven trazados con bellos rasgos y brillantes valores el valor y el patriotismo; elevan el alma, engendran la 
heroicidad, despiertan nobles afectos, y la moral y la virtud recogen en su lectura las más sanas doctrinas. Por último, el latín ha sido la lengua nacional durante muchos siglos; en ella están escritas nuestras primeras historias, nuestras leyes, infinitos actos de las transacciones civiles, y sirve en fin a nuestra religión para celebrar el culto y consignar sus divinos preceptos.

De tal forma que los estudiantes de bachillerato leían la prosa de Cicerón, la historia de Tito-Livio, César o Salustio; los poemas de Horacio, Virgilio y Ovidio; el teatro de Terencio y Eurípides, las fábulas de Fedro y Esopo (Rodríguez Guerrero, C. y Soto Picornell, C., 2006, p. 12), entre otras. Todos estos textos se recopilaban en antologías y colecciones como la Colección de autores selectos latinos y castellanos para uso de los institutos, colegios de segunda enseñanza (1849), o en la Colección de Autores y Trozos selectos para uso en las aulas de Bachillerato, publicada por el Gobierno en 1851, en la que "se incluyen todas las lecturas que un alumno debe realizar en los seis cursos de escolaridad" (Rodríguez Guerrero, C. y Soto Picornell, C., 2006, p. 12) ${ }^{3}$.

En algunas de esas colecciones aparecian, a veces, también autores castellanos, principalmente aquellos que suponian un ejemplo moral y religioso para los alumnos. De ahí que los escritores que más se leían en esta etapa educativa fueran Fray Luis de León, Fray Luis de Granada y, cómo no, Cervantes; y se alejaba a los estudiantes de aquellas lecturas que pudieran dañar su moralidad; de tal forma que no podian entretenerse con el Libro de Buen Amor, o con La Celestina, libros llenos de ejemplos de la vida mundana, a pesar de su categoría literaria. Otro de los autores proscritos fue Góngora; de él dice Campillo y Correa, catedrático del instituto Cardenal Cisneros y autor de una de estas recopilaciones:

No es posible comprender a Góngora sin empezar diciendo, que en él hay dos poetas: el de los romances y letrillas, y el de las Soledades y Polifemos [...]. El primero, correspondiente a su primera época, es natural, inspirado, gallardo, valiente, dominando la expresión de un modo asombroso, no inferior al más excelso de los líricos españoles; el segundo es afectado, artificioso, oscuro, falso en las imágenes y sentimientos y por completo extraviado, hasta rayar en lo absurdo y monstruoso [...]. Aun fueron más deplorables para nuestra literatura sus consecuencias por el gran talento del innovador, que arrastró con su ejemplo a casi todos los escritores de su época, inclusos [sic] Lope de Vega, Calderón y Quevedo, enemigos del gongorismo, pero también finalmente contagiados de su influencia (Campillo Correa, 1885, p. 262) ${ }^{4}$.

Estas lecturas, concienzudamente seleccionadas por su contenido ético, eran las recomendadas a un alumno de bachillerato de las primeras décadas del siglo XX. Uno de ellos fue el escritor y filólogo Alonso Zamora Vicente, que estudió en el instituto de San Isidro, y que recuerda cómo eran aquellas clases en las que el profesor les mandaba leer Corazón de Edmundo de Amicis, en traducción de Hermenegildo Giner de los Ríos. No nos costará mucho imaginar cómo las lágrimas corren por las mejillas del alumno ante las aventuras y peligros que corre el joven Enrique:

Leen cuando no hay nada que hacer, cuando el maestro está cansado, cuando el día negruzco del invierno no ayuda a nada, cuando el tranvía chirría en la cuesta, al pasar bajo el arco de la Plaza Mayor. Se lee para Ilenar el horario. Los colegiales leen Corazón, de Edmundo de Amicis, venga a zamparse heroicidades y viajes infantiles, y reuniones más o menos distinguidas. Qué monótona la voz del maestro, arrullo tenaz bajo la fatiga, mientras va desgranando las aventurillas patrióticas del tamborcillo sardo, del vigía lombardo, subido a su árbol hasta encontrar la muerte, o la actitud quijotesca del patriota paduano, o el larguísimo viaje desde los Apeninos a los Andes... Sí, los chiquillos de entonces estábamos aprendiendo, qué importante, la unidad italiana, la lucha contra el positivismo a fuerza de románticos, despoblada la voz por la rutina, saltándonos los renglones aposta cuando el maestro dormitaba (Zamora Vicente, 1981, p. 3).

\section{La Biblioteca Literaria del Estudiante, una idea de la Junta para Ampliación de Estudios}

Ante esta abundancia de colecciones que no satisfacian la necesidad que tenía el estudiante de conocer y apreciar en conjunto la obra de los grandes autores castellanos, la Junta para Ampliación de Estudios comenzó a publicar en 1922 una selección de lecturas para los alumnos del Instituto-Escuela ${ }^{5}$, experiencia docente para niños de primaria y de secundaria, que se había creado en $1918^{6}$. Con la Biblioteca Literaria del Estudiante, la JAE pretendía ofre- 
cer unos textos adecuados, en extensión y calidad literaria, a las exigencias del nivel educativo de los estudiantes ${ }^{7}$. La creación de esta Biblioteca se fundamenta en tres criterios principales, según se reconoce en la Memoria de la Junta de 1922 (Memoria de la JAE, 1921-22, pp. 278-279)8: la conservación del texto original, es decir, que el alumno tuviera a su disposición las obras tal y como las escribió el autor, sin censuras; una selección hecha en función de su valor filológico, histórico literario y en su interés para los estudiantes; y el tercer objetivo era que los libros tuvieran un precio asequible para todos los estudiantes y sus familias, sin que por ello dejara de ser una publicación atractiva y educadora.

No fue éste el único intento de la Junta para acercar la lectura de los autores clásicos a los estudiantes, pues con las Misiones Pedagógicas se realizó un esfuerzo similar, que tenía el mismo cometido de facilitar libros a las escuelas rurales. El Servicio de Bibliotecas fue tal vez el más importante de los que formaba el Patronato de las Misiones Pedagógicas. Coordinado por el poeta Luis Cernuda y por los bibliotecarios María Moliner y Juan Vicens de la Llave recibía casi un $60 \%$ del presupuesto total. A cada biblioteca escolar se le entregaba una colección de 100 volúmenes, fruto de una selección del patronato que se hacia en virtud del interés que pudieran suscitar a los lectores $^{9}$. Varios de los ejemplares de la Biblioteca Literaria del Estudiante formaron parte de esa selección que distribuía el Servicio de Bibliotecas de las Misiones Pedagógicas por los distintos pueblos.

Otro proyecto que se puso en marcha dentro de la JAE para favorecer la lectura de los estudiantes fue la Biblioteca Circulante para Niños que creó el Museo Pedagógico y que les facilitaba libros de literatura infantil y textos escolares que también contribuyeron al aprendizaje de la lectura y la escritura.

Fuera del ámbito de la Junta, en los primeros años del siglo $X X$, se produjo en España un fuerte crecimiento de la industria editorial, y la publicación de libros creció de forma considerable ${ }^{10}$. De entre los distintos proyectos editoriales interesantes que surgieron, podemos destacar, ya que también se centró en la edición de los autores clásicos, aunque no dedicados sólo a un público estudiantil, la colección Clásicos Castellanos La Lectura. La Lectura (Revista de Ciencias y de Artes) era una publicación mensual creada en 1901 por Clemente Velasco, y dirigida por Francisco Acebal, que se había formado en la Institución Libre de Enseñanza y colaboró con José Castillejo en la Junta para Ampliación de Estudios, de la que fue vicesecretario. Como redactor trabajó primero Julián Juderías y después Domingo Barnés, también muy unido a la Institución y a la Junta, que sustituyó a Cossío en la dirección del Museo Pedagógico, y que fue el impulsor de las grandes colecciones de la editorial: "Ciencia y Educación", dedicada a temas educativos, "Biblioteca Juventud", dirigida a un público adolescente, y "Clásicos Castellanos", centrada en el mundo de la literatura española. Esta última colección se caracterizaba por trasladar a los ejemplares el rigor y la metodología filológica que existía en el Centro de Estudios Históricos, ya que muchos de los responsables de las ediciones eran filólogos colaboradores del propio Centro, principalmente Américo Castro y Navarro Tomás:

La idea respecto a la selección de obras y autores, tipo de comentario en notas y prólogos y hasta tamaño de libro y clase de papel se fue madurando en las reuniones nocturnas que celebrábamos con Acebal, en su casa de la calle de Lista cerca del paseo de la Castellana, Felipe Clemente de Velasco que era el propietario de La Lectura, Américo Castro y yo [...]. El plan era que Castro y yo, que aún no habiamos hecho oposiciones ni ganado plaza, nos dedicáramos plenamente a ir dando cada uno dos o tres volúmenes para la colección (Navarro Tomás en Seris, 1973, p. 257) ${ }^{11}$.

La colección Clásicos Castellanos se proponía difundir nuestra riqueza literaria en volúmenes modernos, aunque no exclusivamente dirigidos a bachilleres, y además lo hacía en ediciones con gran precisión filológica, con introducciones, anotaciones y con un texto lo más cercano posible al original. Como le decía en una carta Américo Castro a Cossio, querían acercarse "a esas viejas prosas con respeto y con amor"12.

Esos tres objetivos son los mismos que se proponía la Biblioteca Literaria del Estudiante. Sin embargo, a pesar de que planteaban ofrecer los textos íntegros para que el alumno pudiera valorar la obra en su conjunto, en algunas ocasiones, debido a su extensión, se tenían que conformar con fragmentos. En estos casos, descartaban aquellas partes que podian resultar más complicadas para la comprensión del joven, pero siempre que el pensamiento del autor no se perdiera, ni su estilo se desfigurara.

ARBOR Vol. 187749 mayo-junio [2011] 547-560 ISSN: 0210-1963

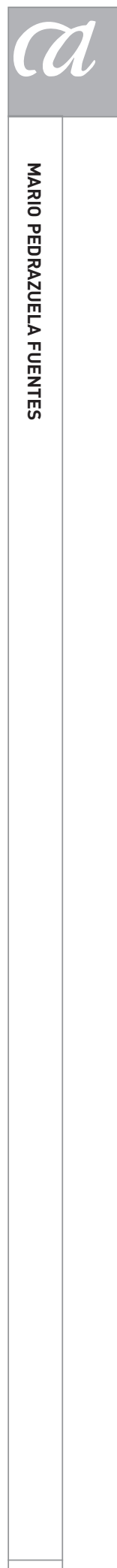

549 
Ramón Menéndez Pidal dirigía la colección y contó con la ayuda inicial de Antonio García Solalinde, colaborador suyo en el Centro de Estudios Históricos, que se encargaba de los trabajos de organización. La Biblioteca constaba de 30 volúmenes que suponen un recorrido por la literatura española de todas las épocas. La organización se hizo en función de dos criterios: por un lado el género, ya que se seleccionan fragmentos de obras de teatro, de novelas, poesía e historia; y por el otro las distintas etapas literarias. Comienza la Biblioteca, aparte de los tres primeros números dedicados a la cultura tradicional, con los ejemplares que tratan la literatura contemporánea, de ellos, uno recopila fragmentos de prosistas, otro poesía, y otro teatro, además de uno dedicado al gran novelista del momento, Benito Pérez Galdós. De los siglos XVIII y XIX, uno de los tomos está consagrado al teatro romántico y el otro a los autores del dieciocho. En los Siglos de Oro se da gran importancia al teatro, con cuatro tomos: Calderón, Ruiz de Alarcón, Tirso de Molina y Lope de Vega; en los volúmenes destinados a la historia se recopiló a los historiadores de los siglos XVI y XVII, así como a los exploradores y conquistadores de Indias, de tal forma que también se ofrecian textos de autores americanos. En la novela no podía faltar Cervantes, al que se le dedican dos libros, uno para El Quijote y otro a sus novelas y teatro. Dentro de la novelistica se ofrece una selección de fragmentos de novelas picarescas, y otra de cuentos de los siglos XVI y XVII. También hay un volumen que recoge una antología de poetas áureos. El último bloque está destinado a la Edad Media y comprende cinco ejemplares que compendian fragmentos del Romancero, de la obra de don Juan Manuel, cuentos medievales, de Alfonso X el Sabio, y de los Cantares de gesta y leyendas heroicas.

Como acabamos de ver, la Biblioteca está formada principalmente por las obras maestras de nuestra literatura, cuyo conocimiento es necesario para que cualquier estudiante de bachillerato adquiera las nociones básicas de la cultura hispánica, a pesar de las dificultades de comprensión que su lectura pudiera acarrear. Para compensar este aspecto, también se introdujeron aquellas obras que, por su carácter elemental y sencillez, se acercaban más a las preferencias y sensibilidad de los adolescentes, pero no exentas de valor artístico o histórico, como son un cancionero musical, una recopilación de fábulas en verso y otra de cuentos tradicionales, que fueron los tres primeros números de la colección. Sin embargo, en la Biblioteca, al igual que sucedia en muchas de las recopilaciones que se hacian en el siglo XIX y principios del $X X$, tampoco aparecieron recogidos algunos títulos imprescindibles de literatura española, como pueden ser el Libro de Buen Amor o La Celestina. De la misma manera que ocurría entonces, el Góngora que se recoge es de los romances y letrillas, y no el de las Soledades o el Polifemo; tampoco interesa el Quevedo satírico, burlón y sarcástico. Tal vez se deban estas ausencias a lo que el director de la colección, Ramón Menéndez Pidal, afirmaba en las páginas preliminares: "La presente Biblioteca trata de incluir en treinta tomitos las obras cuyo conocimiento nos parece más esencial o más oportuno en los primeros años de la enseñanza" (Menéndez Pidal, 1922, p. 2).

Cada uno de los libros llevaba ilustraciones hechas por Fernando Marco, que fue el ilustrador de la primera edición de Platero y yo, que apareció en 1914 en la editorial La Lectura, y muchos de ellos una pequeña introducción en la que se acercaba al joven lector a la obra y al autor que tenía delante. La extensión variaba entre las 150 y las 350 páginas, y su precio podía oscilar entre las dos y las cuatro pesetas, según el número de páginas.

Los destinatarios de estos libritos no eran sólo los estudiantes de bachillerato, también los de la escuela primaria, así como los de universidades, especialmente extranjeras, en las que servían como apoyo al aprendizaje de la lengua y literatura españolas, y así se utilizó en algunas universidades estadounidenses. Aurelio M. Espinosa, profesor de Stanford University, se hace eco de la publicación de la BLE en un artículo publicado en Hispania en 1923, en él hace una pequeña reseña de los tomos publicados hasta entonces ${ }^{13}$. También Cony Sturgis, profesor de Oberlin College, en Ohio, donde formó una importante colección de novelas históricas populares del siglo XIX, publicó en 1928 un artículo sobre las publicaciones que se habian hecho en España en ese año y destaca los tomos de la Biblioteca Literaria del Estudiante: "Over here we start our youngsters of with a "Book of Knowledge" facts and more facts, but this series gives the young Spaniard a picture of the great literature from the Cancionero to Prosistas modernos" (Sturgis, C., 1928, p. 376). 


\section{La Biblioteca Literaria del Estudiante y la EDUCACIÓN DEL GUSTO Y EL SENTIMIENTO ARTÍSTICO}

En todos estos ámbitos educativos, la colección se sitúa dentro del espíritu innovador en el campo de la pedagogía que desarrollaba el Instituto-Escuela en particular, y la Junta para Ampliación de Estudios en general. Según Samuel Gili Gaya, catedrático de Lengua y Literatura en el Instituto-Escuela, la finalidad del Biblioteca Literaria del Estudiante en el campo de la literatura era "proporcionar el conocimiento directo de las obras más importantes de nuestra literatura y de las líneas generales de su evolución histórica" (JAE, 1925, p. 146). Pero además, también se pretendía que estos libros fuesen modelos para avanzar en un futuro de progreso, como decía don Ramón:

Modelos para la imitación que [no] cohiban la nativa frescura del que los estudia, sino que se propone algo más elevado y eficiente: quiere entrañar los principales productos literarios en la inteligencia del lector asiduo, para que el pensamiento y el lenguaje de éste se enriquezca, $y$, devolviéndose con fuerte arraigo en la tradición, tomen como punto de partida el pasado a fin de poder conseguir la línea de progreso que la tradición señala hacia lo porvenir (Menéndez Pidal, 1922, p. 4).

Desde el Instituto-Escuela se propone un nuevo concepto de la enseñanza de la lengua y de la literatura en la que el alumno no tenga que memorizar datos que fatiguen su cerebro, sino que buscan una enseñanza que se centra en transmitir al adolescente, según se recoge en el Reglamento de la institución, "un dominio del idioma como medio fiel y dócil de expresión del pensamiento, y a la formación de un estilo personal y vigoroso; una educación del raciocinio, utilizando análisis lógicos del lenguaje; y una educación del gusto, mediante el conocimiento de obras selectas de las literaturas patrias y extranjera"14.

Para conseguir estos objetivos, en los cursos de primaria, María Goyri ${ }^{15}$ propuso un método de trabajo con los ejemplares de la Biblioteca Literaria del Estudiante, que consistía, para la clase de lectura, en que cada día el profesor disponía los fragmentos que se iban a leer; los alumnos buscaban en el diccionario las palabras que no entendian y las escribian en sus cuadernos en los que formaban un pequeño vocabulario. En la de recitación, el profesor además de elegir el texto, explicaba el valor artís- tico del poema y daba algunas nociones sobre el autor, de esta forma el niño se iba creando una pequeña antología de poemas y de fábulas de diversos autores, así como de romances. En las clases de redacción, el alumno utilizaba los libros de la Biblioteca Literaria del Estudiante primero para hacer un resumen de los textos, y después escribía él mismo algún hecho del que él fuera protagonista, o bien una reflexión sobre su propia labor. En cuanto a la literatura, se proponía que ya desde jovencitos conocieran un ciclo literario determinado, que iba cambiando en virtud de los programas "para evitar que las maestras hagan una enseñanza rutinaria y para que la lección tenga siempre la lozanía de lo recién creado" (de Maeztu, y Goyri de Menéndez Pidal, 1926, p. 412). Así, por ejemplo, proponía la esposa de Menéndez Pidal estudiar el Cid en la historia, en el teatro, en el romancero y en los poemas, y que los ejercicios de vocabulario, de redacción y de recitación se enfocaran hacia este tema; o el cuento tradicional, lo que daría material suficiente para realizar un breve ensayo sobre literatura comparada.

En definitiva, lo que se proponía doña María era otorgar a los alumnos hábitos de expresión escrita del pensamiento en la lengua materna; conocer lo necesario de la gramática para poder utilizar la lengua de forma correcta; despertar el desarrollo de los sentimientos estéticos y de las vocaciones literarias; $y$, por último, proporcionar un conocimiento de las obras más representativas de nuestra literatura y su evolución histórica ${ }^{16}$. La propia María Goyri fue la encargada de editar el primer volumen y número uno de la Biblioteca Literaria del Estudiante, que se publicó en 1922, con el título de Fábulas y cuentos en versos, y el XXVII, dedicado a don Juan Manuel, que salió publicado en 1936.

En bachillerato, Samuel Gili Gaya y Miguel Herrero, que también editaron varios títulos de la colección, como veremos más adelante, fueron los encargados de mostrar los métodos de trabajo que se seguían en las clases de literatura. Las clases tenían como propósito dar hábitos de expresión oral y escrita del pensamiento en la lengua materna; facilitar un conocimiento del mecanismo gramatical necesario para el aprendizaje de otras lenguas; favorecer el desarrollo de los sentimientos artísticos; y proporcionar un conocimiento de las obras más importantes de nuestra literatura y de su evolución histórica. Si nos centramos únicamente en el aspecto literario, la lectura era la base

ARBOR Vol. 187749 mayo-junio [2011] 547-560 ISSN: 0210-1963 
en la que se apoyaban estas clases, y cada año tenían los estudiantes la obligación de leer uno de los tomos de la Biblioteca Literaria del Estudiante ${ }^{17}$, aparte de otras lecturas que mandara el profesor. En los dos primeros cursos el profesor leía fragmentos breves de obras que pudieran despertar la sensibilidad de los alumnos (romances, cuentos, fábulas), y también de aquellos textos más representativos de nuestra literatura (El Cid, Los infantes de Lara, Don Quijote).

A partir del tercer año comienzan las lecturas individuales a las que se dedica una hora semanal. Mientras leen, aquel que ha terminado su lectura, le cuenta en privado al profesor lo que ha leído: sus puntos esenciales, la época, el autor. Tienen la obligación de leer 10 libros, aunque algunos estudiantes "llegaron a dar cuenta al profesor de hasta 40 libros por curso"18. En estos cursos empiezan a ver cuestiones referidas a los géneros literarios, también a la historia de la literatura de forma resumida, así como se les da algunas nociones básicas de gramática histórica a partir de la lectura de un libro que siempre es una obra del Siglo de Oro.

En quinto y sexto curso, comienzan las clases especiales para los alumnos de letras. En estas clases a los estudiantes, además de pedirles que se prepararan en su casa unos cuantos versos del Cantar del Mío Cid para cada día de clase, también se les exigía la lectura de un libro semanal de los siglos XVI y XVII, y que redactaran un impresión crítica acerca de él. Sobre esa misma época se les pedía que hicieran un trabajo de investigación de un tema elegido, por lo que desde el mes de mayo se interrumpían las clases de lecturas. En el último curso, las lecturas se hacian de obras de los siglos XVIII y XIX y autores contemporáneos más importantes. Resulta curioso como en este año se les mandaba comentar seis obras medievales debido a que entendían que el alumno tenía ya un dominio de la lengua medieval suficiente como para leer los textos sin dificultad, por otra parte "la sensibilidad necesaria para deleitarse en la lectura de los primitivos no suele despertar sino después de una intensa cultura literaria"19.

Como vemos, tanto en la primaria como en la secundaria, la lectura comprensiva se convierte en uno de los nuevos pilares de la educación literaria. Ya no se le pide al alumno que memorice poemas o fragmentos de obras, sino que comprenda lo que lee y que esa lectura le sirva por un lado para despertar su conciencia de gusto ante la obra de arte, y por otro le ayude a dominar su lengua, faceta imprescindible para poder ordenar y transmitir con claridad sus pensamientos. La Biblioteca Literaria del Estudiante es un instrumento de gran utilidad para que el profesor pueda conseguir estos objetivos pedagógicos.

\section{LOS EJEMPLARES Y LOS EDITORES DE LA BIBLIOTECA LITERARIA DEL ESTUDIANTE}

No es casual que fuese María Goyri quien iniciara la colección, pues fue ella una de las impulsoras de la Biblioteca. Había participado en la preparación del proyecto de creación del Instituto-Escuela, junto a José Castillejo, María de Maeztu y Luis de Zulueta; además de formar parte del Patronato ${ }^{20}$. Y en esa aventura pedagógica que inició la JAE en 1918, doña María se dio cuenta de que uno de los grandes defectos que tenía la enseñanza, principalmente en los primeros años, que fue en los que ella más se implicó, era la carencia de unos textos que permitieran a los alumnos conocer las fuentes de nuestra literatura y los textos literarios. Como hemos visto más arriba, fue ella, en colaboración con María de Maeztu, la encargada de redactar los programas para la enseñanza de la lengua y literatura españolas para los niños de ocho a diez años del Instituto-Escuela, y entonces se percató de la necesidad de poner a disposición de ellos los mejores textos de la literatura castellana adaptados a sus circunstancias.

Pero no solamente doña María se implicó en el InstitutoEscuela y en la Biblioteca Literaria del Estudiante, sino que toda la familia Menéndez Pidal participó activamente en el proyecto. Desde su creación, don Ramón estuvo presente en la selección del profesorado y presidió su Patronato entre 1928 y 1933, además de dirigir la Biblioteca Literaria del Estudiante. La hija, Jimena, dirigió, a partir de 1933, la sección de párvulos, y editó, dentro de la Biblioteca, un volumen dedicado a la épica. Su marido, Miguel Catalán, fue profesor del centro; y Gonzalo, el hijo menor, además de estudiar en el Instituto, se encargó de la edición del Romancero.

La preocupación de María Goiry por la enseñanza para los más jóvenes, fue la razón por la que los tres primeros 
libros de la colección se dedicaron a la literatura tradicional, a aquellas obras que, como decía don Ramón en la presentación de la Biblioteca, "por su sencillez y carácter elemental cuadran a los primeros años de la vida y de los estudios mejor que otras obras de mayor significación y alcance artístico".

En el primer volumen, titulado Fábulas y cuentos en verso, y que salió publicado en 1922, Maria Goyri, sin seguir un orden cronológico, toma de crónicas, poemas épicos y comedias, una serie de fábulas escritas en verso de autores muy diferentes, esencialmente de Lope de Vega, Tirso de Molina, Antonio Mira de Amescua, Calderón de la Barca, Rojas Zorrilla o José Cañizares, entre otros muchos ${ }^{21}$. Además de la selección, añadió unas notas explicativas y un glosario que aparece al final. También fue la editora, como veremos más adelante, del volumen XXVII, dedicado a don Juan Manuel, que salió publicado en 1936.

Acompañaba al número uno un prólogo escrito por Ramón Menéndez Pidal en el que explica las razones que Ilevaron a la Junta para Ampliación de Estudios a publicar la Biblioteca. En ese prólogo, don Ramón anunciaba que la JAE Ilevaba tiempo trabajando en la colección para darla continuidad y rapidez, y que ya se encontraban preparados gran parte de los textos; de tal manera que en los dos primeros años, entre 1922 y 1923, es decir, antes del golpe de Estado del general Primo de Rivera, vieron la luz 12 tomos. Después el ritmo de las publicaciones descendió: dos en 1924, uno en 1925, tres en 1926, uno en 1928, y de ahí tenemos que saltar a 1933, 1934 y 1936 cuando se publicaron los tres últimos ejemplares. Sin embargo, sí hubo continuas reediciones de los tomos publicados.

En 1933 se reeditó el libro Fábulas y cuentos en verso, y en él, como en la primera edición, don Ramón añadió unas palabras en las que se hace eco del éxito que ha tenido la colección entre los profesores de la escuela y de la segunda enseñanza como guía para sus lecturas en clase.

Junto a la familia Menéndez Pidal, hubo otros editores, todos ellos, a excepción de José Ramón Lomba y Pedraja y Ramón María Tenreiro, relacionados de una manera u otra con el Instituto-Escuela o con la Junta para Ampliación de Estudios, que les pagaba alrededor de unas 1.000 pesetas por la edición de cada tomo ${ }^{22}$.
En aquel primer año de publicaciones, 1922, salieron a la luz ocho títulos diferentes. Uno de los que más éxito tuvo fue el número IV, que era una recopilación de prosistas contemporáneos hecha por Enrique Díaz Canedo, poeta y crítico literario que colaboró estrechamente con la JAE, y que en 1935 dirigió la revista editada en el Centro de Estudios Históricos, Tierra Firme. En este volumen se recogen fragmentos de obras de autores que van desde el romanticismo de Bécquer, al realismo de Pardo Bazán, Clarín, Palcio Valdés, Blasco Ibáñez, Galdós, pasando por Mariano de Cavia, Becerro de Bengoa, José Nogales, para terminar con los de la generación del 98, Unamuno, Valle-Inclán, Pío Baroja y Azorín. También se recopilan fragmentos de obras de autores hispanoamericanos, como Ricardo Palma y José Martí. Fue, tal vez, el título que más reediciones tuvo, en 1922, 1925, 1930, 1933.

Margarita de Mayo fue alumna de Rafael Altamira en el Seminario que daba en el Centro de Estudios Históricos sobre Metodología Histórica e Historia contemporánea, y después, tras haber estado una temporada en Londres como profesora de cursos de elemental y secundaria, fue maestra de la sección de preparatoria del Instituto-Escuela, desde 1918 hasta que en 1924 se marchó a los Estados Unidos a dar clases de español en Vassar College. Ella preparó la selección de las novelas más representativas de Galdós (tomo V), con dos fragmentos largos de Zaragoza, Marianela; y dos más breves de Fortunata y Jacinta y San Vicente de la Barquera.

Samuel Gili Gaya, colaborador de Ramón Menéndez Pidal en el Centro de Estudios Históricos y profesor del Instituto-Escuela, del que dirigió la Sección de Bachillerato del Hipódromo, se encargó de hacer una edición de las obras de Tirso de Molina (tomo XIII), en la que recogió fragmentos de El condenado por desconfiado, El burlador de Sevilla, La prudencia en la mujer y La villana de Vallecas. Al año siguiente, en 1923, don Samuel sería el encargado de hacer la selección para los estudiantes de las obras de Calderón de la Barca; y en 1925 publicó el tomo dedicado a los historiadores de los siglos XVI y XVII (tomo XVI), que dividió en tres grandes bloques: historiadores generales $y$ crónicas de reinados, historiadores de sucesos particulares, e historiadores de Indias.

Otro catedrático del Instituto-Escuela, Juan Dantín Cereceda, que lo fue de Agricultura entre 1919 y 1922, se

ARBOR Vol. 187749 mayo-junio [2011] 547-560 ISSN: 0210-1963

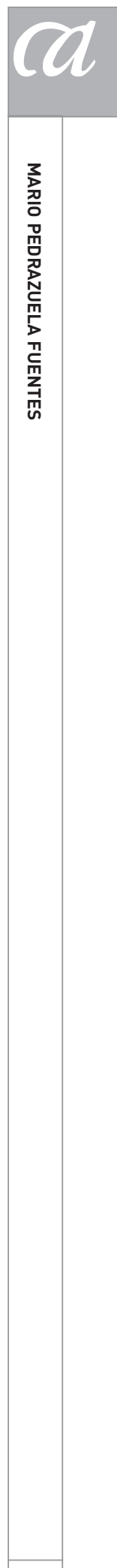

553 
encargó de hacer una antología de los textos de los Exploradores y conquistadores de Indias. Relatos geográficos (tomo XVII). En su selección se fijó principalmente en aquellos fragmentos en los que los conquistadores nos narran las continuas sorpresas que les causan la fauna y la flora de la tierra americana, así como las costumbres de las poblaciones amerindias.

En ese año inaugural de la Biblioteca no podia faltar Cervantes. Se publicaron, como ya hemos dicho, dos tomos. El primero, dedicado a sus novelas y teatro (tomo XXI), fue seleccionado por Josefina Sela, que estuvo becada por la JAE durante un año para estudiar la enseñanza de la lengua y la literatura en las escuelas de primaria y secundaria en Alemania, y que posteriormente fue directora de la Sección de Párvulos y Preparatoria de la zona del Retiro del Instituto-Escuela. En ese volumen recogió fragmentos de las novelas cortas La Gitanilla, La ilustre fregona, Novela y coloquio que pasó entre Cipión y Berganza; y de la novela bizantina Historia de los trabajos de Persiles y Segismundo; asi como de las obras de teatro El retablo de las maravillas, El cerco de Numancia, Pedro de Urdemalas.

De la edición del Quijote (tomo XXII) se encargó José Ramón Lomba y Pedraja ${ }^{23}$, que también fue el editor de otros dos volúmenes. Catedrático de la Universidad de Murcia y de la de Oviedo, fue amigo de juventud de Menéndez Pidal, con quien había compartido aficiones literarias y campestres desde 1890, y que colaboró durante los primeros años del siglo XX en la recolección de romances por la zona de Cantabria; con él recorrió la ruta de los Infantes de Lara.

En 1924, publicó una selección de dramaturgos anteriores a Lope de Vega (tomo XV), entre los que se encontraban Juan de la Encina, Torres Naharro, Gil Vicente, Juan de la Cueva y Lope de Rueda. Ya en 1926, se encargó del volumen dedicado al teatro romántico, época de la que era un especialista porque había escrito un libro relacionado con la figura de don Juan Tenorio y varios sobre la vida y la obra de Larra.

Las publicaciones de ese primer año de creación de la Biblioteca, es decir, en 1922, terminaron con el tomo XXIV dedicado a la novela picaresca a cargo de Federico Ruiz Morcuende, uno de los primeros colaboradores de don Ramón en el Centro Estudios Históricos, oficial del Cuerpo Facultativo de Archivos Bibliotecarios y Arqueólogos con destino en la Biblioteca Nacional, y al que la JAE otorgó una pensión en 1927 para realizar visitas de estudio de ordenación bibliográfica en Inglaterra, Francia y Alemania. Eligió fragmentos de La vida del Lazarillo; de Cervantes, Rinconete y Cortadillo; El buscón, de Quevedo; también del Guzmán de Alfarache, de Mateo Alemán; y por último, una parte del El diablo cojuelo, de Vélez de Guevara.

Como ya hemos dicho, en 1923 el ritmo de publicaciones bajó, aparte del Calderón de Samuel Gili Gaya (tomo XI), con fragmentos de La vida es sueño, El alcalde de Zalamea, El príncipe constante, Casa con dos puertas difícil es de guardar, La puente de Mantible, Cena del rey Baltasar y La viña de Señor, Américo Castro fue el encargado de preparar la edición de las comedias de Lope de Vega (tomo XIV). Don Américo siempre se mostró muy preocupado por el mundo de la enseñanza, tanto secundaria como universitaria, y cuando se crea el Instituto-Escuela envía una serie de recomendaciones a José Castillejo, referidas principalmente a la selección del profesorado ${ }^{24}$. En el prólogo del libro, Castro apuntaba que la Biblioteca aspira a difundir entre los estudiantes "el gusto por las letras españolas y el conocimiento de las lenguas, de la vida y de la historia que aquéllas reflejan"; e indicaba a los jóvenes cómo tienen que acercarse a estos libros: "Sin solemnidad ritual, pero con el mayor arte posible, y haciendo que el alumno se dé cuenta de que maneja textos esenciales de la civilización española, sobre los cuales no puede resbalarse con ligereza". Además de la introducción, Castro escribió unas notas sobre la vida del autor y las obras escogidas, que fueron Amar sin saber a quién, El mejor alcalde, el rey, El caballero de Olmedo y Peribáñez y el comendador de Ocaña.

Jimena Menéndez Pidal se encargó de la publicación del número XXX, en el que selecciona, bajo el título Poema del Cid y otras gestas heroicas, fragmentos de gestas heroicas, entre los que se encuentran Bernardo del Carpio, Poema de Fernán González, Los siete infantes de Lara y Poema de mío Cid. Al final, incluye un vocabulario que facilita la compresión de la lectura; y para situar al lector geográficamente, al principio de cada obra, coloca un mapa en el que se pueden ver los lugares donde sucedieron los hechos que se cuentan, además de abundantes notas.

Pedro Blanco Suárez, profesor de Lengua y Literatura y secretario del Museo Pedagógico, preparó el tomito dedicado a los poetas de los siglos XVI y XVII (tomo XIX), entre 
los que aparecen Garcilaso de la Vega, Gutierre de Cetina, Cristóbal de Castillejo, Fray Luis de León, Francisco la Torre, Fernando de Herrera, San Juan de la Cruz, los hermanos Argensola, Góngora, Lope de Vega, Quevedo, por citar a los más representativos.

Al año siguiente, en 1924, se publicó un tomito dedicado a los libros de caballerías (tomo XX), con selección a cargo de Ramón María Tenreiro, político y escritor. Se recogen fragmentos del Amadís de Gaula y Palmerín de Inglaterra, precedidas de un prólogo. También el dedicado al teatro de la época anterior a Lope de Vega, del que se encargó Lomba y Pedraja, como ya hemos visto.

En 1925 únicamente salió publicado el tomo dedicado a los historiadores, que preparó Gili Gaya. Al año siguiente, en 1926, salieron publicados tres nuevos ejemplares. El teatro romántico (tomo IX) de Lomba y Pedraja, ya indicado $^{25}$, y una recopilación de las obras de Ruiz de Alarcón (tomo XII) hecha por José Vallejo, catedrático de Latín del instituto de Segovia, y desde 1920 agregado al InstitutoEscuela hasta que en 1932 fue nombrado catedrático de Lengua y Literatura latinas en la Universidad de Sevilla. Escoge fragmentos de La verdad sospechosa, Examen de maridos, Las paredes oyen y No hay mal que por bien no venga, a las que antepone una breve introducción con datos biográficos del autor y análisis de las obras. Un año después le fue concedida una beca de la JAE para estudiar métrica y sintaxis latina en Inglaterra. El tercer tomo que salió publicado ese año fue Cuentos de los siglos XVI y XVII (tomo XXIII), por Miguel Herrero, catedrático de Lengua latina del instituto de Cuenca y agregado al InstitutoEscuela, que disfrutó de una beca de la JAE para estudiar en Francia y Suiza la organización de la enseñanza. De esa estancia existe una copiosa correspondencia con José Castillejo en la que realiza diferentes propuestas sobre la enseñanza secundaria ${ }^{26}$. También se le concedió otra beca en 1925 para hacer estudios de lengua y literatura en Inglaterra. En su ejemplar, Miguel Herrero se proponía tres objetivos principalmente: presentar todas las formas en que fue cultivado el cuento en España, recoger el mayor número de autores y de estilos, y no dejar fuera los cuentos consagrados tanto en la literatura española como en el folclore universal.

En 1928 se publicó únicamente el tomo dedicado al Cancionero musical (tomo III), que recoge composicio- nes desde el siglo XIII hasta principios del XX; junto a las canciones se incluye la música que las acompaña. La recopilación está hecha por Eduardo Martínez Torner, colaborador de Ramón Menéndez Pidal en el Centro de Estudios Históricos, principalmente en la recopilación de romances y canciones tradicionales, y que sería el encargado del coro de las Misiones Pedagógicas en la Segunda República.

Durante los años de la Segunda República únicamente se publicaron tres tomos: una colección de romances hecha por Gonzalo Menéndez Pidal en 1933 (tomo XXV), una antología de poetas místicos españoles a cargo de Luis Álvarez Santullano en 1934 (tomo XVIII), y el dedicado a don Juan Manuel del que se encargó María Goyri (tomo XXVII). En el primero, el hijo de don Ramón y alumno del Instituto-Escuela, hace una selección de romances y los clasifica por temas (históricos, fronterizos, moriscos, carolingios novelescos, líricos viejos y de la tradición actual), acompañados de un prólogo y en algunos casos de la música. Por su parte, Santullano, alumno de Cossío, inspector de primera enseñanza, vicesecretario de la JAE y secretario de las Misiones Pedagógicas, recoge poemas de Fray Francisco de Osuna, Fray Alonso de Madrid, Fray Luis de Granada, Teresa de Jesús, Fray Luis de León, Malón de Chaide, Fray Juan de los Ángeles y San Juan de la Cruz. Antecede a la antología un prólogo y una breve noticia sobre la vida de cada uno de los poetas. En estos dos volúmenes ya no aparecen las ilustraciones de Fernando Marco, que son sustituidas, en el primero por las de Antonio Ruiz Castillo, y en el segundo por fotografías de cuadros y de paisajes.

Por último, en 1936, la misma que había iniciado la colección en 1922, María Goyri, la pone fin sin desearlo, ya que la guerra paralizó su publicación. En este volumen titulado Don Juan Manuel y los cuentos medievales, selecciona fragmentos de las obras de don Juan Manuel: El conde Lucanor, Calila y Dimna, El libro de los gatos, El libro de los ejemplos y Las fábulas de Isopete. Las fotografías que acompañan al texto fueron hechas por Gonzalo Menéndez-Pidal. En la advertencia que hace la editora al inicio del libro, detalla los manuscritos y las bibliotecas de donde proceden los fragmentos recopilados, así como las mejores ediciones que existen de esos textos, lo que demuestra el rigor filológico de esta colección.

ARBOR Vol. 187749 mayo-junio [2011] 547-560 ISSN: 0210-1963

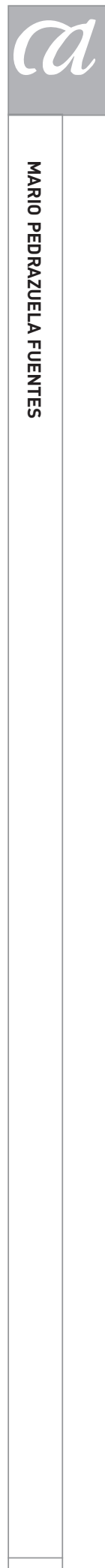

555 


\section{UNA COLECCIÓN INCOMPLETA}

Los libros de la Biblioteca Literaria del Estudiante no sirvieron únicamente para formar a estudiantes españoles, pues además de ser utilizados en universidades de los Estados Unidos, como hemos visto, también en paises iberoamericanos sirvieron en colegios e institutos para familiarizar al estudiante con las obras y los autores clásicos de la literatura hispánica. En 1925, durante un viaje diplomático a Río de Janeiro, el ministro de Estado, que era el propio dictador, don Miguel Primo de Rivera, repartió entre los centros de enseñanza el catálogo de la Biblioteca ${ }^{27}$. También el encargado de negocios del mismo ministerio en Santo Domingo hizo por el país caribeño propaganda de la Biblioteca ${ }^{28}$.

Pero ni los colegiales españoles ni los hispanoamericanos pudieron disfrutar de la colección al completo, pues no se llegó a publicar entera. En las memorias de la JAE únicamente se anuncia la publicación de 22 tomos $^{29}$. De la lista de 30 títulos que se anuncian en el primero de ellos, no se llegó a dar a la imprenta el tomo dedicado a los cuentos tradicionales (II), ni los que recopilaban el teatro y la poesía contemporánea (VII y VIII), ni las piezas de teatro cortas (VI), así como los escritores del siglo XVIII (X), y los cuentos y la poesía medievales (XXVI, XXVIII), tampoco el número XXIX, con la obra de Alfonso $X$ el Sabio. Vemos que algunos de los que no se publicaron estaban dedicados a la literatura más actual, respecto a la que dice don Ramón en el prólogo al primer librito: "Especialmente se notará éstas [las ausencias] en los autores más recientes, pues aquí la falta es siempre menos sensible, por hallarse los libros modernos más al alcance de todos". Parece, pues, que al organizar la colección, dejaron para el final los tomos dedicados a la literatura del momento, de ahí que se quedaran sin publicar. También se podría deber a los problemas que tuvieron con los autores o sus herederos para conseguir los textos, como sucedió con el dedicado al teatro romántico. Los herederos de Duque de Rivas pusieron varios problemas para ceder la publicación del Don Álvaro, como le cuenta el editor de ese tomo, Lomba y Pedraja, a Menéndez Pidal:

Sin duda que la publicación del famoso tomo del Teatro Romántico está por el momento en un pantano del que puede no será fácil sacarla. Y el nudo del asunto está, a mi parecer, en los herederos del Duque de Rivas. Por lo cual concedo importancia a la entrevista que me propongo tener con la Duquesa y con su hermana (que son las propias interesadas) [...]. Cuenta además con que la oferta que podemos hacer (300 ó 400 pts?) no es en modo alguno tentadora para la Duquesa, que es hoy muy rica, y por este lado podemos apretar poco. Mientras esto no esté resuelto, tratar con los demás no urge $^{30}$.

Tampoco todos los editores que se anuncian en ese primer volumen llegan a colaborar con la Biblioteca, pues ni Tomás Navarro Tomás, ni Antonio García Solalinde, ni Justo Gómez Ocerin, dieron a la imprenta ningún ejemplar. Tal vez la razón se deba a que el colaborador que iba a ayudar a Menéndez Pidal en la organización de la Biblioteca Literaria del Estudiante, García Solalinde, se marchó en 1922, un año después de que se creara, a la Universidad de Wisconsin, en los Estados Unidos, para dar clases. No sería de extrañar que él fuera el encargado de editar el volumen dedicado a Alfonso $X$, pues por esos años se encontraba preparando la edición de la General Historia del rey sabio. Don Ramón, con sus innumerables trabajos en la Junta, en el Centro, en la Real Academia de la Lengua, no tenía tiempo para dedicarse a la colección.

Tras la guerra, una vez desaparecida la Junta para Ampliación de Estudios y creado en su lugar el Consejo Superior de Investigaciones Científicas, muchos de los proyectos iniciados por aquélla los retoma éste, entre ellos la Biblioteca Literaria del Estudiante. A pesar de las dificultades para poder mantener unos precios asequibles a todos los públicos, debido principalmente a la carencia de papel, "el CSIC ha procurado conservar esta colección al alcance de la juventud escolar, a la que está dedicado", se nos informa en la solapa.

En los primeros años del franquismo, desaparecieron de entre los editores aquellos que lo habían sido en la época anterior, y sus lugares los ocuparon personas afines al régimen. Desde el CSIC se centraron principalmente en publicar aquellos tomos que no lo habían sido en la etapa anterior. Los primeros fueron las piezas cortas de teatro y el teatro contemporáneo, en 1944 y 1947 respectivamente, con una selección hecha por Eduardo Juliá Martínez. Rafael de Balbín fue el encargado de realizar en 1952 el volumen dedicado a los poetas contemporáneos; Manuel Cardenal Irachea recopiló las obras de Alfonso X, en 1946; Luis González Palencia, el destinado a la poesía medieval 
en 1947. En 1944, Juan Antonio Tamayo volvió a editar el teatro de Tirso de Molina, con lo que desapareció Samuel Gili Gaya de la cubierta del libro ${ }^{31}$. El nuevo editor mantuvo la misma selección que había hecho el anterior. A partir de los años cincuenta y sesenta, cuando el régimen franquista va relajando los postulados falangistas de los primeros años, los antiguos editores vuelven a aparecer en las reimpresiones que se van haciendo.

\section{Conclusiones}

A principios del siglo $X X$, las carencias pedagógicas que existian en España empiezan lentamente a ser resueltas, principalmente debido al esfuerzo iniciado por la Institución Libre de Enseñanza y continuado por la Junta para Ampliación de Estudios. Una de esas carencias era la de facilitar a los estudiantes una selección fiable de las obras más representativas de la literatura española, que superara las viejas colecciones de trozos decimonónicos, en volúmenes de calidad al tiempo que asequibles para la economía familiar. Con este fin se creó la Biblioteca Literaria del Estudiante, que, junto con otros proyectos editoriales, se proponía acabar con el desconocimiento que existía entre los bachilleres de nuestra riqueza literaria.

Esta colección se enmarca dentro de la renovación pedagógica que se propuso llevar a cabo para alumnos de secundaria en el Instituto-Escuela. En el ámbito de la lengua y literatura se buscaba educar el gusto y desarrollar los sentimientos artísticos de los estudiantes, así como ayudarles a conseguir hábitos de expresión oral y escrita de su pensamiento, al tiempo que les proporcionaba un conocimiento de primera mano de las obras más representativas de literatura hispánica, labor en la que desempeñó un papel importante la Biblioteca Literaria del Estudiantes.

Pero de sus ejemplares, además de los estudiantes del Instituto-Escuela, que pertenecian a la élite intelectual de la época, también pudieron disfrutarlos los niños y adolescentes de las aldeas más recónditas de nuestra geografía, ya que muchos de sus títulos formaban parte de la colección que el Servicio de Publicaciones de las Misiones Pedagógicas repartió por los pueblos para que crearan sus bibliotecas. También en Hispanoamérica sirvió para fomentar la educación literaria de los escolares.

Don Ramón Menéndez Pidal, que contó con la ayuda de toda su familia, en especial de su mujer María Goiry, dirigió la colección, en la que colaboraron expertos en cada una de las obras y de los autores seleccionados, casi todos ellos relacionados con el Instituto-Escuela o con la JAE. La vida de la Biblioteca fue breve, ya que como todos los proyectos de la JAE, se vio truncada por la guerra civil. Tras la guerra, el CSIC editó aquellos títulos que no se habían publicado y reeditó algunos de los que ya habían salido a la calle, aunque en algunas ocasiones, los encargados de la edición original desaparecieron de las cubiertas debido a razones de tipo político.

\section{NOTAS}

1 Real Decreto aprobando el plan general de estudios para la instrucción pública del reino en la parte relativa á las enseñanzas secundaria y superior, de 25 de septiembre de 1845, Gaceta de Madrid n. 4029. Se le conoce con el nombre de plan Pidal porque fue aprobado siendo ministro de Gobernación Pedro José Pidal.
2 Real Decreto reformando los estudios de segunda enseñanza y las enseñanzas técnicas del Magisterio, Agricultura, Industria, Comercio, Bellas Artes y Artes industriales, 19 de agosto de 1901, Gaceta n. ${ }^{\circ} 231$. Así lo justificaba en el preámbulo el Ministro: "Se imponía la derogación del absurdo pedagógico, en virtud del cual se mezclaba el estudio de una lengua muerta, como el latín, con el de una lengua viva, como el castellano".
Aceptado: 30 de junio de 2010 
3 Otras recopilaciones son la de Saturnino Fernández (1873), catedrático del instituto de San Isidro, y la de Elias Alfaro y Navarro (1897).

4 Una opinión parecida del mismo autor la recogen Carmen Rodríguez y Carmen Soto (2006, p. 16).

5 "Una colección como esta que ahora se publica, como cosa muy diferente de las 'colecciones de trozos escogidos' que aun bien hechas, nos han parecido siempre completamente ineficaces para formar, no ya el conocimiento de nuestra literatura nacional, sino lo que más importa en la segunda enseñanza [...]: el gusto y la curiosidad por esta literatura." Reseña de H. S. M. (1923).

6 Martínez Alfaro, Encarnación (2009).

7 Alonso Zamora Vicente recuerda cómo fue la llegada de los libritos de la Biblioteca Literaria del Estudiantes a su instituto: "Y ya en bachillerato [...] me surge en el recuerdo la Biblioteca literaria del estudiante, editada por la Junta para Ampliación de Estudios, en la que, por muy poco dinero, nos familiarizábamos con Lope de Vega, Calderón, Alarcón, Lázaro de Tormes...". Alonso Zamora Vicente (1995), p. 33.

8 Memoria de la JAE, 1921-22, pp. 278279.

9 Sobre las bibliotecas de las Misiones Pedagógicas, véanse, entre otros, Mariano Boza Puerta y Miguel Ángel Sánchez Herrador (2004) y Ramón Salaberría (2006).

10 "España estaría de enhorabuena, si todas sus industrias hubiesen realizado los mismos progresos que la editorial en los diez años últimos. Hace dos lustros, nuestros libros del tipo corriente no podian competir en presentación con los extranjeros. Hoy igualan a unos y superan a otros. Compárese cualquier volumen nuestro de tres o cuatro pesetas con los franceses de siete y se verá que los aventaja en el número de páginas, en la calidad del papel y en la impresión. Estos progresos son más sensibles cada dia.". Manuel Ciges Aparicio, "Ayuda, no trabas", El Imparcial, 26 de julio de 1919. Cit. por Victor Fuentes (2006), p. 30.

11 Navarro Tomás inauguró esta colección en 1910 con La moradas de Santa Teresa; al año siguiente publicó las Poesías de Garcilaso de la Vega. Américo Castro publicó El Buscón, de Quevedo y Teatro de Tirso de Molina. Otros colaboradores de la sección de Filología del Centro de Estudios Históricos que participaron en esta colección fueron Federico de Onís, Vicente García de Diego, Justo Gómez Ocerín, Samuel Gili Gaya, Antonio García Solalinde, Pedro Salinas, José Fernández Montesinos, incluso Ramón Menéndez Pidal con una edición del Poema de Mío Cid.

12 "No sé si antes de marcharse le indiqué el proyecto de hacer una biblioteca de clásicos castellanos. El proyecto es ya un hecho, pues está terminándose un tomo de Sta. Teresa -Las Moradas- y empieza a imprimirse un tomo de dos comedias de Tirso - El vergonzoso y El burlador-. Otro discípulo de Pidal [Navarro Tomás] y yo estamos encargados de la edición; consiste ésta en dar los textos bien depurados, sirviéndose de Mss. o de ediciones princeps, y en acompañarlos de una introducción, y de notas gramaticales, históricas o literarias, de modo que el público que lea tales libros sospeche el camino que había que seguir en un estudio de mayor entidad. La idea en sí es excelente, verdad?" 15 de septiembre de 1909, David Castillejo (1997, pp. 593-594). Sobre esta colección véanse: Antonio Marco Garcia (1989) y Luis S. Granjel (1973).
13 Espinosa, A. M. (1923).

14 Artículo 11 del Reglamento del Instituto-Escuela, aprobado el 10 de julio de 1918, Gaceta de Madrid n. ${ }^{\circ}$ 199, de 18 de julio de 1918.

15 María de Maeztu y María Goyri de Menéndez Pidal (1926).

16 Muchas veces, incluso cuando ya no era profesora del Instituto-Escuela, doña María se pasaba por las aulas, como recuerda Julio Caro Baroja: "A las clases venía a hacer una inspección periódica doña María Goyri. Mi madre era amiga de María [de Maeztu] y conocía sólo superficialmente a la mujer de Menéndez Pidal. Mas para mí en la escuela ésta fue más estimulante y me alentó repetidas veces en mi afición a las letras. De vez en cuando añadia alguna explicación a lasque daba la profesora. Era una señora alta, corpulenta, con el pelo gris, y tenía, a mi juicio, un tipo muy vasco" (Caro Baroja, 1972, p. 159).

17 "La publicación de la BLE, que los niños manejan ya desde las secciones preparatorias, ha venido a resolver el problema de tener reunidas en 30 tomos las obras esenciales de la Literatura española. Mientras no esté totalmente publicada habrá que acudir a ediciones corrientes, sin selección, y será forzoso prescindir de obras importantes que contengan capitulos poco adecuados para ser leídos por niños", Junta para Ampliación de Estudios (1925, pp. 149-150). En el primer curso tenía como lectura obligatoria el tomo V, Galdós, en segundo, el tomo IV Prosistas modernos, en tercero Cervantes, Quijote, tomo XXII y en cuarto Cervantes. Novelas ejemplares y teatro, tomo XXI.

18 Ibidem, p. 156.

19 Ibidem, p. 163.

20 Elvira Ontañón (2007).

21 Véase María del Rosario Ozaeta (1998). 
22 Asi, por ejemplo, Samuel Gili Gaya, en 1926, cobró 1.000 pesetas por la edición de Historiadores de los siglos XVI y XVII. Archivo Edad de Plata, Residencia de Estudiantes (Madrid), JAE/68-501. La misma cantidad se le pagó a Lomba y Pedrajas, en 1930, por la edición del Teatro romántico, JAE 86-207. José Vallejo cobró 1.130, en 1927, por el teatro de Ruiz de Alarcón, JAE 146/42.

23 Sobre la edición de este libro le escribe Lomba y Pedraja a Menéndez Pidal: "Ayer 11, recibí tu carta del 7; cuando las pruebas del Quijote, corregidas, debian de estar ya cansadas de estar de vuelta en poder del señor Palenzuela que, en efecto, me las remitió el día 7. El 8 me las dieron, el 9 las puse en el correo y es de esperar que el 10 estuvieran otra vez en la imprenta. Ya ves que no me he dormido. En cuanto a abreviar las lañas, ya no es tiempo. No tengo ya en la memoria el texto para poder saber lo que es indispensable y lo que no lo es, aunque bien sé que si algo sobra será muy poco; y aunque este poco, muy relativamente, esto es, dentro de un criterio extremo y excesivo de avaricia de espacio, lo que no me parece plausible dada la extensión total de las lañas y el carácter diminuto de la letra en que las habéis puesto, el cual, por otra parte, podéis disminuir todavía, si es necesario; que en eso no veo ningún inconveniente". Murcia, 12 de noviembre de 1922. Fundación Ramón Menéndez Pidal, Madrid.

24 Véase David Castillejo (1999, pp. 388391).

25 Parece que en esos años, don Ramón propuso un cambio en el diseño de la Biblioteca: "Como, por lo que me dices, veo que has modificado algo el plan primitivo de la Biblioteca del Estudiante, me parece de precisión que me digas a lo menos a la extensión que puedo dar al tomo de dramaturgos románticos que me tienes encargado. En un tomo entero de la biblioteca de Clásicos Castellanos de La Lectura, que tiene 300 páginas de ordinario, y a veces, más, caben dos comedias enteras. Para meter cuatro o cinco comedias en un tomo de la Biblioteca del Estudiante y no constreñirlas a unos extractos excesivamente magros y desnudos, máxime si el libro ha de ir abundantemente ilustrado, hacen falta, según calculo, lo menos otras 300 páginas, esto es, un tomito como el del Quijote, que te acordarás que también hice yo. ¿Estás conforme con esto? Piensa bien cómo tendrá que ser si hay que meterle en menos [...]. Será bueno también que me pongas un límite, o me des un criterio para ponerle, o me remitas algún tomo que haya salido con ilustraciones de las que deseas para la Biblioteca [...]. No creía que tuvieras prisa en la publicación de los tomos de la Biblioteca del Estudiante y antes bien tenía la impresión de que dabas largas adrede para que fueran vendiéndose los publicados. Pero, sabido, me pongo a preparar mi tomo de románticos con la diligencia posible". Carta de J. R. Lomba y Pedraja a Ramón Menéndez Pidal, Oviedo, 14 de octubre de 1925. Fundación Ramón Menéndez Pidal, Madrid.

26 Véase David Castillejo (1999, pp. 453486).

27 Carta enviada a la JAE desde la oficina de Relaciones Culturales Españolas del Ministerio de Estado: "El Ministro de SM en Río de Janeiro dice a este Departamento en despacho n. 31 de fecha 22 de febrero último lo que sigue: Al tener la honra de acusar recibo de V.E. de la R.O. C.A. I, fecha 29 de diciembre último, cúmpleme exponer respetuosamente al V.E. que he dado cumplimiento a la misma remitiendo a los Centros a quienes pudiera interesar el Catálogo de la Biblioteca Literaria del Estudiante que era anejo a la misma. Lo que de Real Orden comunicada por el Sr. Ministro de Estado transmito a V.E. para su conocimiento y con referencia a la de esa Junta de fecha de 9 de diciembre último. Madrid 26 de marzo de 1926". Expediente JAE/161-245. Archivo JAE, Residencia de Estudiantes, Madrid.

28 Carta dirigida por José Castillejo al Secretario General del Ministerio de Estado: "Tengo el honor de acusar recibo a V.E. de la Real Orden de 15 del corriente relativa a la propaganda que el Encargado de negocios de Santo Domingo hace de la Biblioteca Literaria del Estudiante, quedando esta Junta muy agradecida a las gestiones de dicho funcionario y al interés que el Ministro de Estado viene mostrando a la obra de la Junta. Madrid 22 de abril de 1926". Expediente JAE/161-245. Archivo JAE, Residencia de Estudiantes, Madrid.

$29 \mathrm{Ni}$ en la Biblioteca Nacional, ni en la Biblioteca Tomás Navarro Tomás del Centro de Ciencias Humanas y Sociales del CSIC, ni en la de la Residencia de Estudiantes, ni en la de la Fundación Ramón Menéndez Pidal, ni en la del Instituto Isabel la Católica, antiguo Instituto-Escuela, se encuentran todos los ejemplares. En todas ellas se repiten los mismos volúmenes, pero hay otros que no aparecen. Los ejemplares editados de la Biblioteca Literaria del Estudiante se encuentran digitalizados gracias a una iniciativa del CSIC y se pueden consultar en la mencionada Biblioteca Tomás Navarro Tomás.

30 Oviedo 16 de octubre de 1927. Archivo Fundación Ramón Menéndez 
Pidal, Madrid. Finalmente se llegó a un acuerdo: "El consentimiento de los herederos del Duque para un trozo de la Florinda, te manifiesto que para eso no creo que halles obstáculo alguno en el Sr. Anduaga [Gabriel de Anduaga Eguzquiza, marido de Consuelo, hija pequeña del Duque de Rivas], el cual me propuso repetidamente, en la entrevista que con él tuve en la banca Quesada (frente al Congreso) cambiar el Don Álvaro en el tomito proyectado, por otra obra cualquiera del Duque, a lo cual él no se opondría, porque ninguna, salvo Don Álvaro, producía nada a sus dueños". Carta de J. R. Lomba y Pedraja a Ramón Menéndez Pidal, Oviedo, 28 de octubre de 1927. Archivo Fundación Ramón Menéndez Pidal, Madrid.

31 Recordemos que Samuel Gili Gaya había sido depurado después de la guerra y trasladado al instituto de Torrelavega.

\section{BIBLIOGRAFÍA}

Alfaro y Navarro, Elias (1897): Colección de trozos escogidos de los Autores clásicos latinos, para uso de los alumnos de Segunda Enseñanza, Madrid, Imp. de la Viuda é Hija de G. Fuentenebro, 3. ed. corr. (1905).

Boza Puerta, Mariano y Sánchez Herrador, Miguel Ángel (2004): "Las bibliotecas de las Misiones Pedagógicas", Boletín de la Asociación Andaluza de Bibliotecarios, n. ${ }^{\circ} 74$, pp. 41-51.

Campillo y Correa, Narciso (1885): Florilegio español, Madrid, Librería de Hernando, tomo II.

Caro Baroja, Julio (1972): Los Baroja (memorias familiares), Madrid, Taurus.

Castillejo, David (1999): Epistolario de José Castillejo. Tomo III Fatalidad y Porvenir, 1913-1937, Madrid, Castalia.
De Maeztu, Maria y Goyri de Menéndez Pidal, María (1926): "Enseñanzas y métodos", Escuela moderna, Revista Pedagógica y Administrativa de Primera Enseñanza, n. ${ }^{\circ} 417$, junio, año XXXIV, pp. 408-424.

Espinosa, A. M. (1923): "Biblioteca Literaria del Estudiante", Hispania, vol. VI, pp. 199-201.

Fernández, Saturnino (1873): Novísima colección de piezas escogidas de los clásicos latinos, para uso de los jóvenes que se dedican al estudio del latín, ordenadas y comentadas por don Saturnino Fernández Fernández de Velasco, Madrid, Imp. de Bernardino y Caso, 3. ${ }^{\text {e ed. }}$

Fuentes Victor (2001): La marcha del pueblo en las letras españolas 19171936), Madrid, Ediciones de la Torre, 2. ${ }^{\text {a ed. }}$

Granjel, Luis S. (1973): "Biografía de La Lectura", Cuadernos Hispanoamericanos, 272, pp. 306-314.

Junta para Ampliación de Estudios (1925): Un ensayo pedagógico. El InstitutoEscuela de Segunda Enseñanza de Madrid (organización, métodos, resultados), JAE, Madrid.

H. S. M. (1923): Fábulas y cuentos en verso y La novela picaresca (volúmenes I y XXIV de la Biblioteca Literaria del Estudiante) en La Escuela Moderna, 1 de febrero, p. 159.

Marco García, Antonio (1992): "Propósitos filológicos de la colección Clásicos Castellanos de la editorial La Lectura (1910-1935)", Vilanova, Antonio (coord.), Actas del X Congreso de la Asociación Internacional de Hispanistas, Barcelona 21-26 de agosto de 1989.

Martínez Alfaro, Encarnación (2009): Un laboratorio pedagógico de la Junta para Ampliación de Estudios. El Instituto-Escuela Sección Retiro de Madrid, Madrid, Biblioteca Nueva/CEIMES.
Menéndez Pidal, Ramón (1922): Presentación de la Biblioteca Literaria del Estudiante, JAE, Madrid.

Ontañón, Elvira (2007): "El Instituto-Escuela, una experiencia educativa ejemplar", Circunstancia, año $V$, n. ${ }^{\circ} 14$, septiembre.

Ozaeta, Maria del Rosario (1998): "Los fabulistas españoles (con especial referencia a los siglos XVIII y XIX)", EPOS, n. ${ }^{\circ}$ XIV, pp. 196-205.

Rodríguez Guerrero, Carmen y Carmen Soto Picornell (2006): "Leer, leer, leed: 150 años de lecturas en las aulas de Secundaria", Primeras Noticias. Revista de Literatura, n. 223.

Salaberría, Ramón (2006): "Las bibliotecas de Misiones Pedagógicas: medio millón de libros a las aldeas más olvidadas", en Las misiones pedagógicas, 1931-1936, Madrid, Residencia de Estudiantes, pp. 303-317.

Seris Homero (1973): Guía de nuevos temas de literatura española (ed. D. W. McPheeters), Madrid, Castalia.

Sturgis, C. (1928): "Literary Spain, 1928", The Modern Language Jarnual, pp. 375-385.

Thilliez Bianca (2009): "La Biblioteca Literaria del Estudiante: un proyecto pedagógico y editorial del Instituto-Escuela de Madrid", en El largo camino hacia una educación inclusiva: la educación especial y social del siglo XIX a nuestros días: XV Coloquio de Historia de la Educación, Pamplona-Iruña, 29, 30 de junio y 1 de julio, coord. por María Reyes Berruezo Albéniz y Susana Conejero López, vol. 2, pp. 561-578.

Zamora Vicente Alonso (1982): "Lección inaugural y ponencias", Huelva, Diputación Provincial, Instituto de Estudios Onubenses, 1981 Separata de Actas del Congreso Juan Ramón Jiménez, pp. 1-12.

Zamora Vicente, Alonso (1995): La otra esquina de la lengua, Madrid, Universidad Antonio de Nebrija. 